\title{
Prognostic Significance of COX-2 and VEGF in Breast Cancer Patients Undergoing Neoadjuvant Chemotherapy
}

\author{
Gang-Ping WANG ${ }^{1, a,{ }^{*}, \text { Xue WANG }}{ }^{2}$, Zhao-Jun DING ${ }^{3}$, \\ Yun-Ai LIANG ${ }^{1}$, Zuo-Feng ZHANG $^{4}$ \\ ${ }^{1}$ Department of Pathology, Rizhao People's Hospital, Affiliated Jining Medical \\ University, Rizhao, P.R.China \\ ${ }^{2}$ Department of Ultrasound, Rizhao People's Hospital, Rizhao, P.R.China \\ ${ }^{3}$ Department of Oncology, Rizhao People's Hospital, Rizhao, P.R.China \\ ${ }^{4}$ Department of Breast Surgery, Rizhao People's Hospital, Rizhao,P.R.China \\ aemail:wgprzph93@126.com \\ ${ }^{*}$ Corresponding author
}

Keywords: Breast carcinomas, COX-2, VEGF, Immunoreactions, Prognosis.

\begin{abstract}
In recent years, the incidence of breast cancer is signifcantly on the rise all over the world, hence, how to treat breast cancer, correctively evaluate the prognosis and find the postoperative recurrence of patients with breast cancer have been paid attention by more and more scholars all over the world. Biomarkers such as Cyclooxygenase-2 (COX-2) and vascular endothelial growth factor (VEGF) in tissue can be used as prognostic factors in many malignancies. To analyze the relationship between the expressions of COX-2 and VEGF and clinicopathological features in breast cancer, we examined the expressions and clinical significance of COX-2 and VEGF in breast carcinom tissue $(\mathrm{n}=128)$, and their relationships with chemosensitivity in breast carcinoma $(\mathrm{n}=52)$. The study demonstrated that before and after chemotherapy, the expression of COX-2 had changed significantly from $68.75 \%$ down to $33.56 \%$ $(P<0.05)$. Before neoadjuvant chemotherapy, the chemosensitivity of COX-2 positive expression was worse than the chemosensitivity of that nagetive expression $(P<0.05)$, for VEGF, there was no differences significantly $(P>0.05)$. The over-expression of COX-2 and VEGF might be important biological markers for invasion and metastasis of breast carcinomas. COX-2 can be used as predictors of breast cancer chemosensitivity to help develop individualized chemotherapy.
\end{abstract}

\section{Introduction}

In recent years, neoadjuvant chemotherapy has additionally become an option for patients with operable tumors who desire breast conservation therapy. Neoadjuvant chemotherapy with agents such as doxorubicin and cyclophosphamide had historically been offered to patients with locally advanced disease with a goal of reducing tumor size to enable surgical resection. Several randomized clinical trials have demonstrated no statistically significant difference in disease-free survival or overall survival in the patients receiving preoperative therapy as opposed to those receiving postoperative chemotherapy [1]. In recent years in China, the incidence of breast carcinoma is relatively high, and the peak incidence is in advance, a large number of patients died of breast carcinoma complications or serious organ metastasis each year. 
Breast carcinoma, like most other forms of malignancy, is mainly a multifactorial disease, occurring as a result of the combined effects of environmental and heritable factors $[2,3]$. Many studies have found that certain biological indicators by detecting abnormal expression of molecules, such as estrogen receptor (ER), progesterone receptor (PR), proto-oncogene HER2/neu, etc, can guide clinical diagnosis and treatment activities prognosis [4]. The ER, PR and HER2/neu profile of breast carcinoma plays a significant role in patient management and treatment. Because of the increasing utilization of neoadjuvant chemotherapy or hormone therapy, surgicallyresected carcinomas often show marked treatment effect. There are several other molecular biomarkers, such as cyclooxygenase-2 (COX-2) and vascular endothelial growth factor (VEGF) and have been confirmed to participate in the evolution of breast carcinoma [4-6]. COX-2 is an inducible enzyme that interferes with tumor development and angiogenesis, related to the inhibition of apoptosis through inhibition of the proapoptotic Bax protein and over-expression of the antiapoptotic bcl-2 protein. It has been investigated in several human cancers and also correlated with the evolution of the disease [7-10]. VEGF capable of promoting angiogenesis exerts an important effect in the process of genesis, development, metastasis and recurrence of various tumors. Over expression of COX-2 and VEGF is a frequent feature of malignant disease and it is commonly associated with poor prognosis and resistance to conventional chemotherapy. So far, there is no evidence for efficacy of screening with serum biomarkers and tissue markers in breast cancer. To validate whether combined detaction cancer biomarkers in breast cancer tissues and biomarkers in serum may improve accuracy and sensitivity of predicting metastasis and prognosis of breast cancer, and to observe the expressions and clinical significanc of COX-2 and VEGF in breast cancer tissue, we composed a panel of potential biomarker COX-2 and VEGF in breast cancer tissues, in order to explore the above markers expressions and clinicopathological parameters in breast cancer, and study their expressions with chemosensitivity in breast carcinoma.

\section{Patients and Methods}

\section{Patients}

Permission was obtained from the Local Ethical Committee to collect breast cancer tissues and all patients signed informed consents to the research. One handrened twenty-eight patients diagnosed breast cancer were collected during excision surgery at Rizhao people's Hospital from June 2000 to June 2013. Fifty-two cases of breast cancer patients were treated by the clinical protocol as neoadjuvant chemotherapy. Data concerning ER, PR, HER2/neu, COX-2 and VEGF status, and histological grade were recorded. Fifty cases of benign leision were selected as a control group (shown in Table 1). No significant difference was noted regarding general data such as gender, age, and body weight, indicating good comparability between the observation group and control group $(P>0.05)$. The expression of COX-2 and VEGF in breast cancer were detected and their relationship with clinical-pathological parameters including the histological grade, region lymph node metastasis, distant metastasis and recurrence on files were also assessed in order to study the clinical and pathological characteristics associated with breast cancer and improve the clinical diagnosis of breast cancer, monitor whether the cancer was indeed in regression due to the anti-cancer treatment, or reoccurring. Immunohistochemical method was used to detect differences in tumor tissue COX-2 and VEGF expression and the situation before and after chemotherapy in 52 cases of 
breast cancer patients to neoadjuvant chemotherapy. Patients assessed for the expression of ER, PR, HER2/neu, COX-2 and VEGF were grouped as positive or negative. Concerning the histological grade, patients were classified into two groups: low grade (I \& II) and high grade (III).

\section{Histopathology Study}

The pathological diagnosis was verified by histological methods independently by two pathologists, and pathological categorization was determined according to the current WHO classification system (2012). The pathological reading was determined for each biopsy slide with an overall pathological diagnosis determined for each subject. The tumor grade was obtained according to the modified Bloom-Richardson score. In this study, patients were classified into two groups: low grade (I \& II) and high grade (III).

\section{Immunohistochemistry}

Immunohistochemical Ultra Sensitive ${ }^{T M} S-P$ method (Maixin-Bio, Fuzhou, Fujian, China) was used according to the manufacturer's instructions to detect differences in tumor tissue COX-2 and VEGF expression. For COX-2 and VEGF, the immunoreactive score was obtained by multiplying the percentage of positive cells and the staining intensity. In our study, for ER or PR, tumors showing expression in $>10 \%$ of cancer cells were considered positive (not 1\%). ER and/or PR positivity was considered combined ER and PR positivity, and combined ER and PR negativity was considered both ER and PR negativity.

\section{Evaluate Therapeutic Effect}

Immunohistochemical method was used to detect differences in tumor tissue COX-2 and VEGF expression and the situation before and after chemotherapy in 52 cases of breast cancer patients to neoadjuvant chemotherapy. According to the International Union against Cancer (UICC) TNM classification of solid malignant tumors standard (7th ed) and American Joint Committee on Cancer (AJCC) Cancer Staging Manual (7th ed) to evaluate curative effect of therapeutic effect. Complete remission (CR): all known lesions disappeared at least up to 4 weeks; Partial response (PR): measurable lesions, the total volume by $50 \%$ at least more than 4 weeks and no progression or other lesions; stable condition (SD): one or more of the measurement volume reduced less than $50 \%$ of the lesions or increased less than $25 \%$, the time for at least four weeks; Disease progression (PD): one or more measurable lesion volume increases more than $25 \%$ or the emergence of new lesions. The total effective rate was CR and PR.

\section{Statistical Analysis}

SPSS version 17.0 statistical software (SPSS Inc.: Chicago, IL, USA) was used to analyze the data. Enumeration data with chi-squared $\left(\chi^{2}\right)$ test. The relationship of this dichotomous variable to other clinicopathological correlates was established using $\chi^{2}$ or Fisher's exacttests, as appropriate. Kaplan-Meier overall survival curves were constructed to demonstrate the survival differences between the COX-2 positive and negative patients. All other statistical tests were performed using Graphpad Prism 5.0 (Graphpad Software Inc., San Diego, CA). A $p$ value less than 0.05 were deemed statistically significant. 


\section{Results}

\section{Clinical Features}

In A series of 128 cases of women carrying breast cancer was evaluated. A summary of the results concerning major clinicopathological parameters, including pathological tumour stage, nodal status, hormone receptor status, tumour size, grading and vascular space involvement is provided in Table 1.

Table 1. Clinicopathological characteristics of patients

\begin{tabular}{|c|c|c|}
\hline Category & Breast cancer & Benign lesion \\
\hline Number of cases & 128 & 50 \\
\hline \multicolumn{3}{|l|}{ Age at enrollment, Years } \\
\hline Mean \pm SD & $47.5 \pm 5.3$ & $46.2 \pm 5.1$ \\
\hline \multicolumn{3}{|l|}{ body weight(kg) } \\
\hline Mean \pm SD & $65.8 \pm 14.7$ & $65.3 \pm 16.7$ \\
\hline \multicolumn{3}{|l|}{ COX-2, number (\%) } \\
\hline positive & $101(78.91)$ & $8(16.0)$ \\
\hline negative & $27(21.09)$ & $42(84.0)$ \\
\hline \multicolumn{3}{|l|}{ VEGF statuse, number (\%) } \\
\hline positive & $88(68.75)$ & $11(22.0)$ \\
\hline negative & $40(31.25)$ & $39(78.0)$ \\
\hline \multicolumn{3}{|l|}{ Tumor size, number $(\%)$} \\
\hline$\leq 5 \mathrm{~cm}$ & $109(85.16)$ & - \\
\hline$>5 \mathrm{~cm}$ & $19(14.84)$ & - \\
\hline \multicolumn{3}{|c|}{ Histoligical grade, number $(\%)^{*}$} \\
\hline Low-grade & $96(75.00)$ & - \\
\hline High-grade & $32(25.00)$ & - \\
\hline \multicolumn{3}{|l|}{ Vascular invosion } \\
\hline Present & $52(40.63)$ & - \\
\hline Absent & $76(59.37)$ & - \\
\hline \multicolumn{3}{|c|}{ Lymph node metastasis, number $(\%)$} \\
\hline Present & $83(64.84)$ & - \\
\hline Absent & $45(35.16)$ & - \\
\hline \multicolumn{3}{|c|}{ Distant metastasis, number $(\%)$} \\
\hline Present & $37(28.91)$ & - \\
\hline Absent & $91(71.09)$ & - \\
\hline \multicolumn{3}{|l|}{ Recurrence, number $(\%)$} \\
\hline positive & $22(17.19)$ & - \\
\hline negative & $106(82.81)$ & - \\
\hline \multicolumn{3}{|c|}{ Vascular invosion, number $(\%)$} \\
\hline Present & $52(40.63)$ & - \\
\hline Absent & $76(59.37)$ & - \\
\hline \multicolumn{3}{|l|}{ ER statuse, number (\%) } \\
\hline positive & $108(84.38)$ & - \\
\hline negative & $20(9.38)$ & - \\
\hline \multicolumn{3}{|l|}{ PR statuse, number $(\%)$} \\
\hline positive & $110(85.94)$ & - \\
\hline negative & $18(14.06)$ & - \\
\hline \multicolumn{3}{|l|}{ HER2 statuse, number (\%) } \\
\hline positive & $33(25.78)$ & - \\
\hline negative & $95(74.22)$ & - \\
\hline
\end{tabular}




\section{COX-2 and VEGF Express Relation with Clinicopathological Factors}

Immunohistochemical analysis revealed COX-2 and VEGF were weekly expressed only $16.3 \%$ and $22.0 \%$ in benign tissues, however, in the cancer tumors examined, they were some degree of positivity of $78.9 \%$ and $68.75 \%$ (Fig. 1A), the positive rates and the degree of COX-2 and VEGF in breast carcinomas were higher than those in benign tissues $(p<0.05)$ (Fig. 2), and there were difference between high grade (III) and low Grade (I or II) groups (Fig. 1B, C). Relationship between COX-2 and VEGF and clinical-pathological parameters of breast cancer was shown in Fig 1B,C. Statistical analysis revealed significant relationships between COX-2 and VEGF expression and clinical parameters such as histological grade (low $v$ s high) ( $P=0.004, P=0.001$ ), lymph node metastasis $(P=0.000, P=0.006)$, distant metastasis $(P=0.006, P=0.006)$ and TNM stage(I+II vs III $+\mathrm{IV})(P=0.008, P=0.000)$. However, the expressions of the COX-2 and VEGF were not related with age at diagnosis $(\leq 50 \mathrm{yr} v s 50 \mathrm{yr})$, size of tumor $(\leq 5 \mathrm{~cm}$ vs $>5 \mathrm{~cm})$ F.R PR and HF.R $?$ status $(P>005$ resnectivelv)
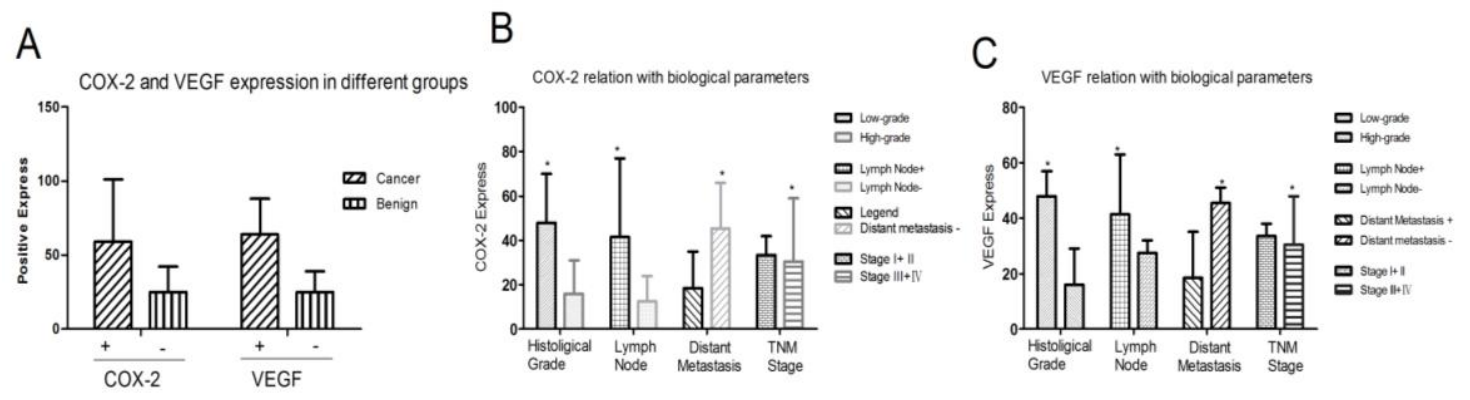

Figure 1. COX-2 and VEGF express and their relationship with clinicopathological factors. (A) comparison between carcinoma and benign lesion, (B) COX-2 and (C) VEGF express relation with biological parameters in breast cancer.

A

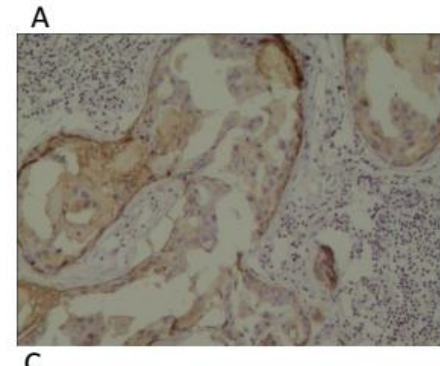

C

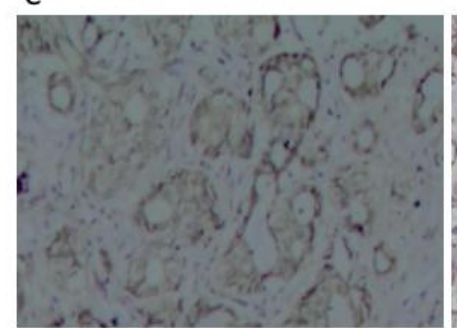

B

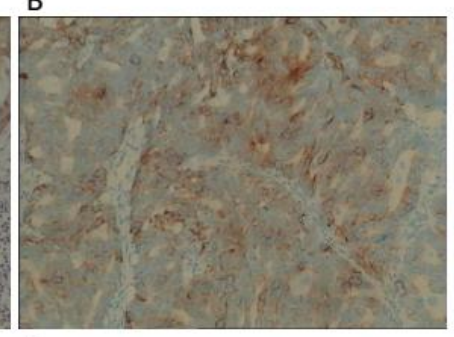

D

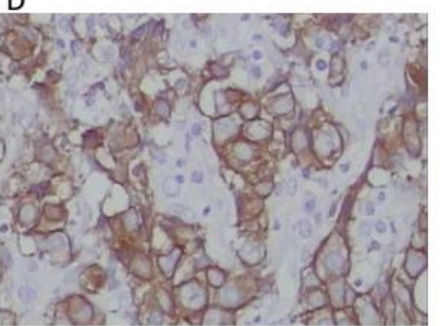

Figure 2. Immunohistochemical of COX-2 expresse in dififfrent breast tissue. COX-2 weekly positive reactivity in cytoplasmic in breast ductal carcinoma in situ (A), intraductal papillary carcinoma (B), low-grade carcinoma (C) and strong intensity expressed cytoplasmic in high-grade carcinoma (D).

Table 2. COX-2 express relation with biological parameters in breast cancer

\begin{tabular}{llllll}
\hline \multirow{2}{*}{ VEGF } & $\mathrm{n}$ & \multicolumn{2}{c}{ COX-2 } & \multirow{2}{*}{$\chi^{2}$} & \multirow{2}{*}{$P$ Value } \\
\cline { 3 - 4 } Positive & 88 & 78 & 10 & 9.441 & $<0.01$ \\
Negative & 40 & 23 & 17 & & $<$ \\
\hline
\end{tabular}


As shown in Table 2, COX-2 in VEGF positive in breast cancer patients was significantly higher than those in negative in breast cancer patients, the expression of VEGF was correlated positively with COX-2 in breast carcinoma $(P<0.05)$.

\section{COX-2 and VEGF Express with Chemotherapy}

ER, PR, HER2, COX-2 and VEGF expression and their situation before and after chemotherapy in 52 cases of breast cancer to neoadjuvant chemotherapy was studied. Our results shown of the 52 carcinomas studied, 37 cases $(71.75 \%)$ were positive for ER by immunohistochemistry both pre- and post- neoadjuvant chemotherapy treatment $(P>0.05)$. The studies showed positivity for PR $36(69.23 \%)$ cases pre-neoadjuvant therapy and $25(48.10 \%)$ positivity post-treatment $(P<0.05)$. For HER2, $16(30.77 \%)$ were positive pre-treatment, and $11(21.15 \%)$ were positive post-treatment. Before and after chemotherapy, the expression of COX-2 has decreased significantly (76.92\% vs $40.38 \%), P<0.05$, for VEGF, there was no significence(69.23\% vs $59.61 \%)(P>0.05)$. The study demonstrated that before and after chemotherapy, the expression of PR and COX-2 had decreased significantly $(P<0.05)$. We considered complete remission $(\mathrm{CR})$ and partial response (PR) for the total effective rate. As shown in Table 3, the chemosensitivity of COX-2 positive expression $(28 / 40,70 \%)$ was worse than the chemosensitivity of COX-2 nagetive expression $(10 / 12,83.33 \%)(P<0.05)$. There was no significent diffrence of chemosensitivity between VEGF positive and negative groups. COX-2 can be used as a predictor of breast cancer chemosensitivity to help develop individualized chemotherapy.

Table 3. Chemosensitivity in biomarker positive and negative groups $(n=52)$

\begin{tabular}{|c|c|c|c|c|}
\hline Biomarker & Number & $\mathrm{CR}+\mathrm{PR}$ & $\mathrm{SD}+\mathrm{PD}$ & Efficient $(\%)$ \\
\hline \multicolumn{5}{|l|}{$\overline{\mathrm{COX}-2}$} \\
\hline Positive & 40 & 28 & 12 & 70.00 \\
\hline Negative & 12 & 10 & 2 & 83.33 \\
\hline \multicolumn{5}{|l|}{ VEGF } \\
\hline Positive & 36 & 26 & 10 & 72.22 \\
\hline Negative & 16 & 12 & 4 & 75.00 \\
\hline
\end{tabular}

Abbreviations: $\mathrm{CR}$, complete remission; $\mathrm{PR}$, partial response; $\mathrm{SD}$, stable disease; $\mathrm{PD}$, progressive disease;

\section{Survival Analysis}

86 case patients were monitored for survival from 2000 to 2014 through telephone communication and periodic returns to the rizhao people's hospital. Considering only the COX-2 expression, survival was found to be longer in negative group than that in positive group, and there were slightly statistically significant between $95 \% \mathrm{CI}=$ $1.715-2.843$, the two groups $\left(\chi^{2}=9.567, P=0.0448\right)$. Kaplan-Meier survival curves demonstrated survival differences between COX-2 positive and negative patients (Fig.3). Other factors may be associated with COX-2 in breast cancer, which should be investigated in further studies. 


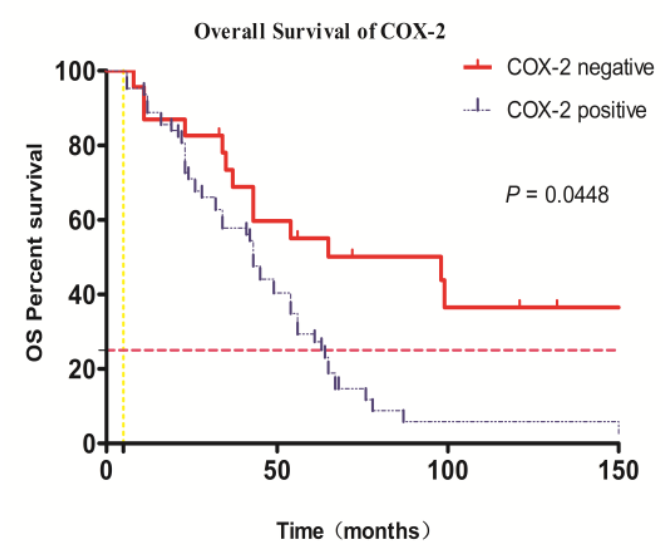

Figure 3. Kaplan-Meier overall survival curves of the patient population under study.

\section{Discussion}

In recent years in China, the incidence of breast carcinoma is relatively high, and the peak incidence is in advance, a large number of patients died of breast carcinoma complications or serious organ metastasis each year [3, 11]. Breast carcinoma, like most other forms of malignancy, is mainly a multifactorial disease, occurring as a result of the combined effects of environmental and heritable factors [6, 12-14]. As a result, numerous studies worldwide have sought to determine the most effective ways to treat breast cancer, assess the rapeutic effects, correctly evaluate prognosis, and identify postoperative recurrence in patients $[1,3,10]$. Many studies have found that certain biological indicators by detecting abnormal expression of molecules, such as ER, PR, HER2/neu, etc, can guide clinical diagnosis and treatment activities prognosis. Because of the increasing utilization of neoadjuvant chemotherapy or hormone therapy, surgically-resected carcinomas often show marked treatment effect. There are several other molecular biomarkers, such as VEGF and COX-2 have been confirmed to participate in the evolution of breast carcinoma [15].

COX-2 is an inducible enzyme that interferes with tumor development and angiogenesis [9-10]. During cancer progression, it participates in arachidonic acid metabolism, generating prostaglandins that can mediate various mechanisms, including cellular proliferation, apoptosis, immune system modulation, and angiogenesis [16]. COX-2 catalyzes the conversion of arachidonic acid to PGE2 and enhances the metastatic phenotype of both breast cancer cells in vitro and breast tumors. PGE2, the catalytic product of COX-2, may promote tumor development and angiogenesis [6, $8-9,16]$. It has been investigated in several human cancers and also correlated with the evolution of the disease $[8,16]$. In our study, in benign leision tissues, only $15.4 \%$ was COX-2 weekly expressed, and some degree of positivity of COX-2 in breast carcinomas are higher than in benign leision tissues. There was positive correlation in over-expression of COX-2 in breast carcinomas with histological high-grade (III), lymph node metastasi and distant metastasis. Our results revealed that patients with increased COX-2 expression have shorter survival times. A strong correlation between COX-2 expression and prognostic factors in breast cancer suggesting that increased COX-2 expression was related to worse prognosis, as observed in the survival analysis in our study. We suggest that the difference between COX-2 expression in the breast carcinomas related to the differentiated behavior of these tumors, confirming the association between the COX-2 expression and disease aggressiveness. Before and 
after chemotherapy, the expression of COX-2 has decreased significantly (76.92\% vs $40.38 \%$ ). The chemosensitivity of COX-2 positive expression (70\%) was worse than the chemosensitivity of COX-2 nagetive expression (83.33\%). Besides showing shorter overall survival, it was verified that COX-2 expression correlated positively with breast carcinoma, suggesting a worse prognosis. In our study, we have observed shorter survival in patients whose tumors expressed more COX-2. Therefore, there is possibility of using COX-2 inhibitor anti-inflammatory drugs to treat breast tumors. COX-2 can be used as a predictor of breast cancer chemosensitivity to help develop individualized chemotherapy.

VEGF, one of the key factors to promote tumor angiogenesis and with the strongest function and highest specificity, can not only promote the proliferation of endothelial cells, but also regulate and participate in angiogenesis [9]. VEGF capable of promoting angiogenesis exerts an important effect in the process of genesis, development, metastasis and recurrence of various tumors [9]. In the process of tumor genesis and development, tumor regenerative capillaries capable of providing nutrients for tumor cells and favorable conditions for distal metastasis are the precondition to induce the local growth, infiltration and distal metastasis of malignant tumors, hence, how to inhibit tumor angiogenesis is a new research hotspot at present. In the study, VEGF expression in different pathological stagings of patients in observation group was analyzed. The results revealed that with pathological staging increasing, the levels of VEGF in observation group gradually increased, and the statistical significance was remarkably presented by comparison $(P<0.01)$. There was significant difference regarding VEGF expression in different pathological stagings of patients $(P>0.05)$. In the study, VEGF expression in the patients with and without lymph node metastasis was compared in observation group. There was posive correlation in over-expression of VEGF with histological grade and lymph node metastasis and distant metastasis of breast cancer, and the expressions of VEGF were not related with age and size of tumor $(P>0.05)$. VEGF, which promotes angiogenesis and promotes the proliferation of endothelial cells, also exerts an important effect in the genesis, development, metastasis, and recurrence of various tumors. In this study, the positive VEGF rate in recurrence group were obviously higher than in non-recurrence group $(P<0.01)$. The result suggest that detection of VEGF indicator above can judge the prognosis better, which is of great importance to monitor recurrence and metastasis. The study demonstrated that before and after chemotherapy, the expression of VEGF was no significence decreased $(P>0.05)$, there was no significent diffrence of chemosensitivity between VEGF positive and negative groups.

\section{Conclusion}

Take integrated analysis of the above results, and the following conclusions can be drawn: COX-2 and VEGF abnormally increased in breast cancer correlates with poor clinicopathological parameters such as invasion and metastasis, related to worse prognosis and shorter overall survival. Before neoadjuvant chemotherapy, the chemosensitivity of COX-2 positive expression is worse than the chemosensitivity of those nagetive expressions, for VEGF, there was no difference being significant. The over-expression of COX-2 and VEGF might be important biological markers for invasion and metastasis of breast invasive carcinomas. COX-2 can be used as predictors of breast cancer chemosensitivity to help develop individualized chemotherapy. 


\section{Conflict of Interest}

The authors declare no conflicts of interest.

\section{Acknowledgements}

This research was financially supported by the Medicine and Health Care Science and Technology Development Plan Project Foundation of Shandong Province (No. 2014WS0282, 2014WSA11003), the Project of Application Technology Research and Development Project Foundation of Rizhao (No. 2014SZSH02), as well as the Scientific Research Key Projects of Jining Medical University (No. JY2013KJ051).

\section{References}

[1] Kinsella1 MD, Nassar A, Siddiqui MT, Cohen C. Estrogen receptor (ER), progesterone receptor (PR), and HER2 expression pre- and post- neoadjuvant chemotherapy in primary breast carcinoma: a single institutional experience.Int $\mathrm{J}$ Clin Exp Pathol 2012; 5 (6):530-6.

[2] Kabalar ME, Karaman A, Aylu B, Ozmen SA, Erdem I. Genetic alterations in benign, preneoplastic and malignant breast lesions. Indian J Pathol Microbiol 2012; 55 : 319-25.

[3] Wang G, Qin Y, Zhang J, Zhao J, Liang Y, Zhang Z, et al. Nipple Discharge of CA15-3, CA125, CEA and TSGF as a New Biomarker Panel for Breast Cancer. Int. J. Mol. Sci.2014;15:9546-65.

[4] Witteveen A, Kwast AB, Sonke GS, Ijzerman MJ, Siesling S. Survival after locoregional recurrence or second primary breast cancer: impact of the disease-free interval. PLoS One 2015; 10:e0120832.

[5] Diers AR, Vayalil PK, Oliva CR, Griguer CE, Darley-Usmar V, Hurst DR, Welch DR , Landar A. Mitochondrial Bioenergetics of Metastatic Breast Cancer Cells in Response to Dynamic Changes in Oxygen Tension: Effects of HIF-1 $\alpha$. PLoS ONE 2013; 8: e68348.

[6] Kim MJ, Kim HS, Lee SH, Yang Y, Lee MS, Lim JS. NDRG2 controls COX-2/PGE2-mediated breast cancer cell migration and invasion. Mol Cells 2014; 37: 759-65.

[7] Lakhani SR, Ellis IO, Schnitt SJ, et al. WHO classification of tumours of the breast[M]. Lyon, France: IARC Press, 2012: 1-117.

[8] Thorat D, Sahu A, Behera R, et al. Association of osteopontin and cyclooxygenase- 2 expression with breast cancer subtypes and their use as potential biomarkers. Oncol Lett. 2013, 6: 1559-64.

[9] Yi C, Zhang Y, Yu Z, Xiao Y, Wang J, et al. Melatonin Enhances the Anti-Tumor Effect of Fisetin by Inhibiting COX-2/iNOS and NF-kB/p300 Signaling Pathways. PLoS ONE 2014, 9 (7) : e99943. doi:10.1371/journal.pone.0099943.

[10] Aggarwal A, Al-Rohil RN, Batra A, et al. Expression of integrin $\alpha 3 \beta 1$ and cyclooxygenase-2 (COX2) are positively correlated in human breast cancer. BMC Cancer. 2014;14:459. doi:10.1186/1471-2407-14-459. 
[11] Wang GP, Zhang H, Zhang ZF, et al. Clinical and pathological characteristics of intraductal proliferative lesions and coexist with invasive ductal carcinomas. Chinses-German J Clin Oncol 2013; 12: 574-80.

[12] Witteveen A, Kwast AB, Sonke GS, Ijzerman MJ, Siesling S. Survival after locoregional recurrence or second primary breast cancer: impact of the disease-free interval. PLoS One2015; 10:e0120832.

[13] Majmundar AJ, Wong WJ, Simon MC. Hypoxia inducible factors and the response to hypoxic stress. Mol Cell 2010;40: 294-309.

[14] Piura E, Piura B. Autoantibodies to tumor-associated antigens in breast carcinoma. J. Oncol 2010; 2010: 264926.

[15] Thielemann A, Baszczuk A, Kopczyński Z, Kopczyński P, Grodecka-Gazdecka S. Clinical usefulness of assessing VEGF and soluble receptors sVEGFR-1 and sVEGFR-2 in women with breast cancer. Ann Agric Environ Med 2013;20: 293-7.

[16] Yu KR, Lee JY, Kim HS, Hong IS, Choi SW, Seo Y, Kang I, Kim JJ, Lee BC, Lee S, Kurtz A, Seo KW, Kang KS. A p38 MAPK-Mediated Alteration of COX-2/PGE2 Regulates Immunomodulatory Properties in Human Mesenchymal Stem Cell Aging. PLoS ONE 2014; 9 : e102426. 